\title{
¿Es útil la monitorización con espectroscopia de infrarojo cercano en la cirugía artroscópica de hombro con anestesia regional y sedación?
}

\author{
Is near-infrared spectroscopy (NIRS) monitoring useful for pa- \\ tients undergoing shoulder surgery under regional anaesthe-
} sia and sedation?

Diego Guardabassi ${ }^{1}$ Laura álvarez , Florencia Bolla', Aníbal Vescovo ${ }^{1}$, Maximiliano Ranelletta ${ }^{1}$

\begin{abstract}
Background: Shoulder surgery in beach chair position is a very common procedure in our daily practice. It has been associated to regional cerebral saturation impairment detected by near-infrared spectroscopy (NIRS) under general anaesthesia. Severe neurological complications were previously reported, even in previously healthy patients. An anaesthetic protocol under regional anaesthesia and biespectral index (BIS) guided sedation seems to be a safer strategy in order to prevent complications. Objective: To find out, in a group of patients undergoing shoulder surgery in beach chair position under regional anaesthesia and sedation, the prevalence of cerebral desaturation events detected by NIRS (defined as a decrease $\geq 20 \%$ from baseline or absolute value $<55 \%$ ). Material and Method: In this descriptive study, 30 patients undergoing shoulder surgery in beach chair position under regional anaesthesia (ultrasound guided interescalene brachial plexus block) and propofol infusion BIS guided sedation were enrolled to assess the prevalence of cerebral desaturation events. The baseline data for regional cerebral oxygen saturation and bispectral index and non invasive blood pressure measured at heart level were taken prior to surgery after beach chair positioning and thereafter all 5 min until discharge. Results: No cerebral desaturation events were detected during this study. Conclusions: Although
\end{abstract}

\section{Key words:}

Shoulder surgery, regional anaesthesia, sedation, regional cerebral saturation, neurological complications

Servicio de Anestesiología. Hospital Italiano de Buenos Aires. Argentina.

Fecha de recepción: 17 de mayo de 2018

Fecha de aceptación: 23 de junio de 2018

ORCID

https://orcid.org/0000-0002-5224-757X

Correspondencia:

Diego Guardabassi

Teléfono: 541163375398.

Los Andes 1837. Haedo (1706). Buenos Aires. Argentina.

Email: guardabassidiego@hotmail.com 
monitoring regional cerebral saturation with NIRS detects decreased cerebral perfusion allowing a rapid intervention, we consider it is not essential under this anaesthesia regimen, considering that no cerebral desaturation events were reported.

\section{RESUMEN}

La cirugía de hombro en posición de sentado constituye un procedimiento frecuente en la práctica diaria. Bajo anestesia general, se ha asociado con caída de la saturación regional cerebral de oxígeno $\left(\mathrm{SrCO}_{2}\right)$ detectada por espectrospcopia infraroja (NIRS), reportándose complicaciones neurológicas severas, incluso en pacientes previamente sanos. El empleo de una técnica de anestesia regional más sedación titulada con índice biespectral (BIS) parecería ser una estrategia más segura a fin de evitar dichos eventos. Objetivo: Conocer, en pacientes sometidos a artroscopia de hombro en posición de sentado bajo anestesia regional más sedación, la prevalencia de episodios de desaturación cerebral (ECDs) determinada por $\mathrm{NIRS}\left(\mathrm{SrCO}_{2}\right.$ inferior a $55 \%$ o disminución del $20 \%$ respecto al basal). Material y Método: Se realizó un estudio descriptivo en 30 pacientes sometidos a artroscopía de hombro en posición de sentado bajo anestesia regional (bloqueo interescalénico ecoguiado) más sedación titulada (infusión de propofol guiada por BIS). Se tomaron valores de referencia de $\mathrm{SrCO}_{2}$, BIS y presión no invasiva a nivel del corazón luego del posicionamiento y, consecutivamente en forma continua para las primeras dos variables y cada 5 minutos hasta el final para la última. Se ocultaron a los anestesiólogos los valores de $\mathrm{SrCO}_{2}$. Resultados: No se ha detectado ningún EDC en la población estudiada. Conclusión: Consideramos que el monitoreo de la perfusión cerebral con tecnología NIRS resulta útil aunque no imprescindible bajo este protocolo anestésico, dada la incidencia casi nula de eventos.

\section{Palabras clave:}

Artroscopía de hombro, anestesia regional, sedación, saturación regional cerebral de oxígeno, complicaciones neurológicas

\section{Introducción}

a cirugía artroscópica de hombro constituye un procedimiento cada vez más habitual en nuestra - práctica diaria. Aproximadamente, dos tercios de estos procedimientos se realizan con el paciente en posición de sentado (silla de playa). Entre las ventajas de esta posición $\left(45^{\circ}-90^{\circ}\right.$ sobre el plano horizontal) se incluyen: un riesgo reducido de estiramiento del plexo braquial y de trauma vascular comparado con la posición en decúbito lateral, excelente visualización intraarticular y mayor facilidad de abordaje en el caso de tener que recurrir a una conversión a cirugía abierta[1]-[2]. Se ha descrito, en numerosas publicaciones, alteraciones en la perfusión cerebral con caída de la saturación cerebral de oxígeno $\left(\mathrm{SrCO}_{2}\right)$ detectadas por NIRS en esta posición quirúrgica bajo anestesia general[2]-[3]-[5]. Aunque en escaso número, fueron informados casos catastróficos de complicaciones neurológicas asociados a dichos procedimientos, como: pérdida de visión, infarto cerebral, estado vegetativo persistente y muerte, ocurridos aún en pacientes jóvenes y sin antecedentes previos[2]-[5]-[6]. La etiología de los mismos no fue aclarada en forma completa, aunque se presume que estaría relacionada con episodios de hipoperfusión cerebral e isquemia intraoperatorios. El manejo de la presión de perfusión cerebral y su autorregulación parecería ser la clave. Esta se halla alterada en pacientes previamente hipertensos y añosos, teniendo en cuenta, además, que sus mecanismos regulatorios son inhibidos por los fármacos anestésicos generales. Si a este último punto se le añade un error hidrostático en la lectura de la tensión arterial (medida habitualmente en el miembro superior contralateral) y éste no es corregido por la diferencia de altura respecto al territorio vascular cerebral, el riesgo de hipoperfusión parece a priori aumentar en forma exponencial[7]-[8].

La espectroscopia de infrarrojo cercano constituye un método no invasivo que registra en forma con- 
tinua la $\mathrm{SrCO}_{2}$. Recientemente, estudios publicados demostraron su utilidad en la detección precoz de desaturación cerebral en pacientes sometidos a cirugía de hombro en posición de sentado bajo anestesia general[8]-[9]-[10]-[11]. Otros trabajos compararon la incidencia de episodios de desaturación de oxígeno cerebral (ECDs) en la posición de sentado vs decúbito lateral, aunque también bajo anestesia general. La incidencia de los mismos es elevada en el primer grupo, de hasta un alarmante $80 \%$ [6]. Existe en la literatura un estudio que compara los ECDs en cirugía de hombro en posición de sentado con anestesia general vs anestesia regional y sedación. Éste demostró una incidencia mayor (estadísticamente significativa) de dichos episodios en el primer grupo, siendo éstos muy poco frecuentes y de corta duración cuando sólo se empleaba anestesia regional y sedación[12].

Se condujo la presente investigación con el objetivo primario de conocer, en pacientes sometidos a artroscopia de hombro en posición silla de playa bajo anestesia regional más sedación titulada por BIS, la prevalencia de episodios de desaturación de oxígeno cerebral detectados por tecnología NIRS, describir sus características y su posible correlación con los antecedentes comórbidos de la población en estudio y con el resto de las variables intraoperatorias medidas.

\section{Material y Método}

El presente estudio fue diseñado como un estudio descriptivo en 30 pacientes sometidos a cirugía artroscópica programada de hombro en posición de silla de playa bajo anestesia regional más sedación profunda (Figura 1).
Se establecieron como criterios de inclusión: pacientes sometidos a cirugía artroscópica de hombro en posición de silla de playa en forma programada; mayores de 18 años. Y como criterios de exclusión: pacientes con enfermedad neurológica previa; enfermedad de vasos de cuello (oclusión carotidea significativa y/o enfermedad de territorio vertebral); embarazo; alergia conocida a los anestésicos locales; trastornos psiquiátricos o del comportamiento.

Después de presentado el protocolo y obtenida la aprobación del Comité de Docencia e Investigación y del Comité de Bioética del Hospital Italiano de Buenos Aires, se dio inicio a la Investigación. La misma se llevó a cabo en nuestra Institución, entre enero de 2015 y diciembre de 2016. Se incluyeron a 30 pacientes a las cuales se les realizó una artroscopia de hombro en forma programada.

El proceso de selección se basó en un muestreo de tipo no probabilístico por serie de casos consecutivos. Éste se efectuó a través de la Sección de Anestesia Regional del Servicio de Anestesiología del Hospital Italiano de Buenos Aires en la sala de estancia pre quirúrgica, donde se entregó el correspondiente formulario de consentimiento informado y se brindaron las explicaciones necesarias respecto al estudio y se resolvieron las dudas que se suscitaron. No fue considerada necesaria la presencia de un testigo.

\section{Se estableció el siguiente protocolo:}

En la sala de recuperación anestésica, luego de comprobar la identidad del paciente y efectuar la marcación del miembro a operar, se colocó un acceso intravenoso en el miembro superior contra lateral. Se reforzó la explicación de la técnica anestésica y monitorización. Se realizó un interrogatorio de rutina,

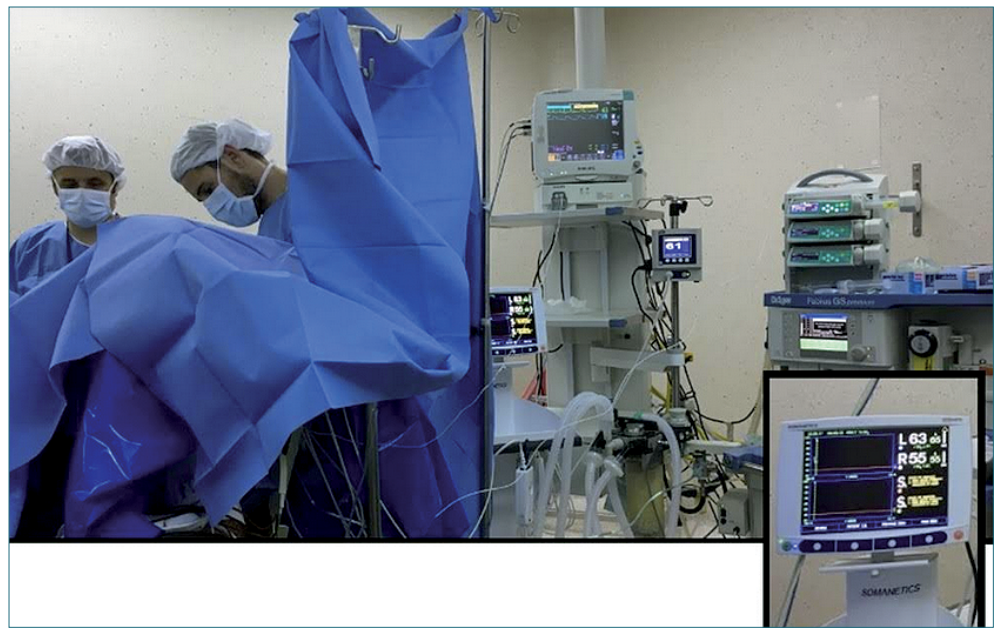

Figura 1. NIRS 19.37.16. 
registrándose antecedentes y datos del examen físico. Se administró midazolam 0,02 mg/kg iv y cefazolina 2 $\mathrm{g}$ iv (si había alergia conocida, se utilizó clindamicina $600 \mathrm{mg}$ iv).

Se estableció una monitorización estándar. Se procedió a realizar un bloqueo del plexo braquial interescalénico guiado por ecografía con transductor lineal de entre 6 a 13 Mhz (ecógrafo Sonosite MTurbo; Sonosite Inc, Bothell, WA 98021, USA). Se utilizó una aguja Pajunk de 50 mm (Pajunk; Germany) insertada con un abordaje ecográfico en plano bajo estrictas normas de antisepsia. Se inyectaron $20 \mathrm{~mL}$ de solución anestésica local (ropivacaína 0,375\% y lidocaína $1 \%$ ) alrededor de los troncos primarios del plexo bajo visión directa.

Después de un tiempo de latencia mínimo de 10 minutos, se trasladó al paciente a quirófano.

Se estableció la siguiente monitorización: saturación de oxígeno continua durante la cirugía; ECG dos derivaciones (DII-V5) (registro continuo); tensión arterial no invasiva, medida en el miembro superior contralateral (se registró el valor basal y cada 5 minutos); registro de temperatura timpánica (basal y cada 15 minutos); capnografía (gafas nasales-registro continuo); índice biespectral (BIS; registro continuo); NIRS: se obtuvo un registro basal y luego continuo durante la cirugía, pero con el monitor cubierto para no ser visualizado por el anestesiólogo a cargo (Figura 2).

Todos los registros basales se realizaron al posicionar al paciente en silla de playa a $75^{\circ}$ y previo a la administración de cualquier fármaco anestésico.

Como protocolo anestésico se empleó: una sedación profunda con infusión continua de propofol al $1 \%$ modalidad TCI (target controlled infussion) titulada para mantener un grado de hipnosis medido por índice biespectral de entre 50 y 70; se mantuvo la tensión arterial media en un valor $\pm 20 \%$ respecto del basal del paciente. En caso de superar el $20 \%$ del mismo, se aumentó la sedación y ante su persistencia, se administró labetalol 10 mg ev o infusión de nitroglicerina. En el caso opuesto, se administró un bolo de solución de Ringer Lactato de $500 \mathrm{~mL}$ y de persistir, se infundió fenilefrina a una dosis de 0,1-0,5 ug/kg/ min; se administró oxígeno mediante gafas nasales para mantener una saturación de oxígeno mayor o igual a 92\%; se monitorizó la ventilación mediante capnografía, interviniendo de ser necesario en caso de obstrucción de la vía aérea/apnea; se colocó una manta térmica con dispositivo de administración de calor externo.

Al finalizar la cirugía, el paciente fue trasladado a la Unidad de Recuperación Anestésica acompañado por el anestesiólogo.

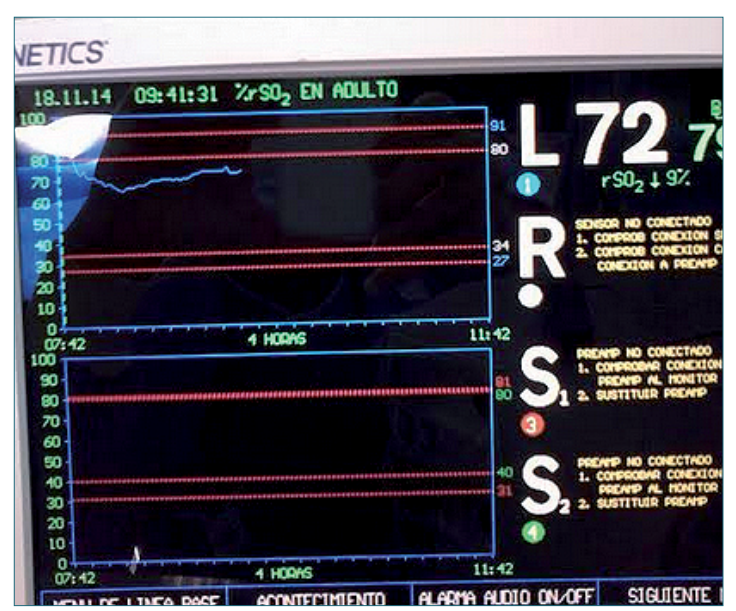

Figura 2. NIRS.

Todos los datos fueron volcados a una planilla informatizada destinada a tal fin. Los datos provenientes del registro continuo los dispositivos BIS y NIRS, fueron analizados mediante el software correspondiente y volcados a una planilla para su posterior análisis estadístico.

\section{Cálculo del tamaño muestral}

Las publicaciones previas reportan en promedio una caída del 10\% de valor de saturación cerebral medido por espectroscopia de infrarrojo cercano (NIRS) respecto al basal en pacientes bajo anestesia regional sometidos a cirugía de hombro. En base a este dato, se efectuó el cálculo del tamaño muestral requerido para detectar una mínima diferencia del $10 \%$ del valor de saturación cerebral entre el valor basal y los obtenidos con el paciente anestesiado en posición de sentado, con un error alfa de 0,05 y un poder de $90 \%$. Se determinó entonces un tamaño de la muestra de 28 pacientes, a los cuales se les sumó un $10 \%$ para cubrir posibles pérdidas.

\section{Análisis estadístico}

El análisis estadístico será realizado utilizando un programa estándar (Stata). La normalidad de los datos fue analizada mediante el test de KolgomorovSmirnov. Los datos normalmente distribuidos son presentados como media \pm desviación estándar, los nonormalmente distribuidos como mediana (rango) y los categóricos como data cruda y frecuencias. Los datos de saturación cerebral de oxígeno en distintos puntos del tiempo fueron comparados usando un análisis de 
varianza para mediciones repetidas (ANOVA) incluyendo la corrección de Bonferroni. Un valor de $p<$ 0,05 fue considerado como significativo.

\section{Resultados}

Treinta pacientes fueron incluidos en la investigación. No se registraron incumplimientos del protocolo ni hubo pérdidas en el seguimiento, por lo que todos los datos pudieron ser analizados.

Respecto a las características de la población, la mediana de la edad fue de 53 (21-74). El 70\% (21 pacientes) eran de sexo masculino. Un 60\% (18 pacientes) presentó al menos 1 factor de riesgo cardiovascular: un 30\% eran hipertensos, 10\% diabéticos, $20 \%$ fumadores, un tercio de los mismos tenían un BMI mayor de 30 y 2 pacientes padecían de enfermedad coronaria. Las mismas se presentan en la Tabla 1.

No se registró ningún episodio de desaturación cerebral medida con tecnología NIRS en nuestra población en estudio. Como criterio de definición del mismo, se consideró una caída de la saturación regional cerebral por debajo de 55\% y/o de un 20\% respecto de la basal del paciente.

Respecto a los objetivos de mantenimiento intraoperatorios, es de destacar que en ningún caso se registraron episodios de hipotensión (definida como una caída de la tensión media por debajo del 20\% de su valor basal). Las variables medidas y registradas durante la cirugía y sus valores promedio son presentados en la Tabla 2.

No hubo ningún episodio clínico de complicación neurológica en el posoperatorio. El confort del paciente respecto a la técnica anestésica empleada no fue evaluado.

Debido a estos hallazgos, al no registrarse ECDs, no pudimos establecer ningún tipo de asociación estadística.

\section{Discusión}

Las complicaciones neurológicas asociadas a cirugía artroscópica de hombro en posición de silla de playa son poco frecuentes, pero pueden ser devastadoras[4]-[11]. El mecanismo de producción parecería estar relacionado con una caída en la presión de perfusión cerebral y consecuentemente, una falta en la disponibilidad celular de oxígeno tanto a nivel central como medular. La autorregulación cerebral se halla alterada en pacientes hipertensos, la reactividad al $\mathrm{CO}_{2}$ disminuye con la edad y la mayoría de los me-

\begin{tabular}{lc}
\hline \multicolumn{2}{c}{$\begin{array}{c}\text { Tabla 1. Características de la población estu- } \\
\text { diada. Factores de riesgo }\end{array}$} \\
\hline Características & $\begin{array}{c}\text { Población } \\
\text { estudiada } \\
\text { (n = 30) }\end{array}$ \\
Sexo (masculino/femenino) & $21 / 9$ \\
Mediana de edad, años (rango) & $53(21-74)$ \\
Hipertensión arterial, \% (n pacientes) & $30 \%(10)$ \\
Diabetes mellitus, \% (n pacientes) & $10 \%(3)$ \\
Fumadores, \% (n pacientes) & $20 \%(6)$ \\
BMI mayor de 30, \% (n pacientes) & $33 \%(10)$ \\
Enfermedad coronaria, \% (n pacientes) & $6,7 \%(2)$ \\
\hline
\end{tabular}

\section{Tabla 2. Variables de registro intraoperatorio.} Valores promedio

\begin{tabular}{|c|c|}
\hline Variable en estudio & Valores promedio \\
\hline $\begin{array}{l}\text { Saturación de oxígeno (\%), } \\
\text { mediana (rango) }\end{array}$ & $97(92-100)$ \\
\hline $\begin{array}{l}\text { Tensión arterial media } \\
(\mathrm{mmHg}) \text {, media } \pm \text { desviación } \\
\text { estándar }\end{array}$ & $78 \pm 15$ \\
\hline $\begin{array}{l}\text { Temperatura timpánica }\left({ }^{\circ} \mathrm{C}\right) \text {, } \\
\text { mediana (rango) }\end{array}$ & $36,2(35,9-36,7)$ \\
\hline $\begin{array}{l}\text { Índice biespectral, mediana } \\
\text { (rango) }\end{array}$ & $56(48-75)$ \\
\hline $\begin{array}{l}\text { Saturación regional cerebral } \\
\text { de oxígeno }(\%) \text {, mediana } \\
\text { (rango) }\end{array}$ & $74(61-82)$ \\
\hline $\begin{array}{l}\text { Caída de la } \mathrm{SrCO}_{2} \text { respecto al } \\
\text { basal (\%), mediana (rango) }\end{array}$ & $8(2-11)$ \\
\hline
\end{tabular}

canismos compensadores son atenuados o inhibidos por el efecto vasodilatador de los agentes anestésicos generales. En adición a estos factores, el error hidrostático en la lectura de la tensión arterial (medida habitualmente en el miembro superior contralateral) no corregido por la diferencia de altura respecto al territorio vascular cerebral, y sobre todo si se emplea un protocolo de hipotensión controlada para la cirugía, parece aumentar en gran medida los riesgos anteriormente citados[13].

Teniendo en cuenta este contexto y sumado a que todos los eventos neurológicos severos reportados fueron bajo anestesia general, resulta interesante el análisis de este grupo de pacientes sometidos al mismo procedimiento quirúrgico con anestesia regional y sedación titulada y la incidencia de eventos 
de desaturación cerebral medidos por NIRS durante el mismo. Siguiendo un estricto protocolo donde destacan el mantenimiento de la tensión arterial dentro de un rango $\pm 20 \%$ del registro basal del paciente (medida en el miembro superior contralateral) y la titulación de la sedación guiada por BIS, no registramos ningún ECD en los 30 pacientes analizados. Este hallazgo puede deberse, en primera instancia, al cuidado estricto, medición correcta y mantenimiento de los valores de tensión arterial dentro de lo establecido. Esto concuerda con los hallazgos de Tange et al., quienes no encontraron ninguna alteración en la saturación cerebral de $\mathrm{O}_{2}\left(\mathrm{SrCO}_{2}\right)$ en posición de silla de playa, con la característica de que en el estudio se mantuvo la presión arterial media (medida a la altura del corazón) entre 75 y 85 mmHg, mediante el empleo de una infusión de fenilefrina[5]. En segundo lugar, el protocolo anestésico empleado basado en el bloqueo regional y sedación titulada, probablemente haya incidido en forma positiva al evitar las alteraciones que generan los fármacos anestésicos generales sobre la tensión arterial media y sobre todo, los mecanismos reguladores de la misma. Al respecto, Yadeau et al., estudiaron el efecto de la anestesia regional con sedación con propofol en la oxigenación cerebral en 99 pacientes sometidos a cirugía de hombro en silla de playa utilizando un protocolo de hipotensión controlada medida a nivel del corazón. A pesar de que encontraron una alta incidencia de hipotensión (99\%), los ECDs fueron poco frecuentes (10\%), sugiriendo un efecto positivo de este tipo de anestesia sobre la perfusión cerebral[11]. Consideramos que el empleo de hipotensión controlada pudo contribuir a la aparición de ese porcentaje de eventos. En apoyo a este último concepto, Koh et al., compararon anestesia general con sevofluorano con anestesia regional con sedación con propofol en 60 pacientes en silla de playa, pero sin el empleo de hipotensión controlada. Su estudio mostró una incidencia de 56,7\% de ECDs en primer grupo y $0 \%$ en el grupo de anestesia regional con sedación[10]. A su vez, Aguirre et al., analizaron 90 pacientes operados de hombro en posición sentada, comparando anestesia regional y general. Sus hallazgos fueron que los pacientes bajo anestesia regional presentaron en forma significativa menos ECDs, una mayor estabilidad hemodinámica en posición de sentado e interesantemente, mejores resultados en las pruebas neuroconductuales en el día posterior a la cirugía[12].

Respecto a la asociación de los ECDs y los factores de riesgo de los pacientes, Salazar et al., encontraron en una investigación una asociación positiva entre un BMI mayor o igual a 34 y los mismos[14]. En otra publicación, Aguirre et al., demostraron una correlación positiva, aunque débil, entre la presencia de factores de riesgo cardiovascular (hipertensión arterial y enfermedad coronaria) y la incidencia de ECDs. No encontraron tal asociación respecto al BMI[12]. Debemos destacar que, a pesar de que nuestra población presentó en un 60\% al menos 1 factor de riesgo cardiovascular, no pudimos establecer ninguna correlación dada la nula incidencia de tales eventos bajo este protocolo anestésico. Esto refuerza la idea de que la anestesia regional preserva los mecanismos de compensación hemodinámica, fundamentales sobre todo en presencia de comorbilidades asociadas.

El mantenimiento de la presión arterial media dentro del rango establecido ( $\pm 20 \%$ de la basal), sumado a que no se registraron episodios de hipotensión en la población en estudio, es un factor determinante para mantener la perfusión regional cerebral, hecho señalado en investigaciones previas. En éstas, McCulloch et al., y Soeding et al., analizaron el flujo sanguíneo cerebral en posición de sentado y describieron una disminución del mismo relacionada con la caída de la presión arterial media, evidenciando una alteración en la autorregulación cerebral. Sin embargo, cuando se mantuvieron los valores de presión estables, el flujo cerebral no se vio afectado[7]-[8].

Consideramos que nuestra investigación, desde lo metodológico, fue objetivamente correcta, con un tamaño muestral adecuado y representativo, un protocolo anestésico estricto, bien definido y aplicado y un registro y evaluación que siguió los estándares institucionales. Las características basales, en cuanto a edad y antecedentes patológicos de la población en estudio, son similares y comparables con investigaciones previas. Otro punto destacado, es que los valores de $\mathrm{SrCO}_{2}$ fueron enmascarados al anestesiólogo, restando de esta manera la posibilidad de intervenir a ese nivel.

Sin embargo, consideramos existen varias limitaciones en nuestro trabajo. En primer lugar, se trata meramente de una investigación descriptiva en un número limitado de pacientes, bajo un protocolo estandarizado empleado en forma rutinaria en nuestra Institución desde hace varios años. Hubiese resultado interesante un formato prospectivo comparativo con un grupo de pacientes bajo anestesia general. El factor limitante respecto de esto último fue meramente ético, ya que tal como fue señalado anteriormente, desde hace ya mucho tiempo realizamos este tipo de cirugías bajo esta estrategia anestésica convencidos de que se trata del estándar de cuidado más seguro para nuestros pacientes y con mejores resultados. En segundo lugar, no se empleó ningún tipo de evalua- 
ción mediante un test neurocognitivo o de comportamiento en el período perioperatorio, lo cual hubiese incorporado un dato más que interesante al análisis global. Tercero, si bien el valor umbral más conocido en la literatura extrapolada de estudios en animales para definir desaturación regional cerebral es por debajo del $65 \%$, establecimos nuestro valor objetivo basándonos en la definición empleada en los estudios previos más representativos y de adecuada calidad metodológica realizados en humanos (caída de la saturación regional cerebral por debajo de 55\% y/o de un $20 \%$ respecto de la basal del paciente)[6]-[12]. Finalmente, y debido al diseño empleado, las conclusiones que pudieron obtenerse no tienen un peso estadístico importante en la toma de decisiones. Sin embargo, creemos que deberían ser tomadas como un aporte más a la extensa bibliografía descripta en la literatura que apoya el empleo de esta estrategia anestésica y que sirven de punto de partida de mejores diseños e investigaciones futuras, en las cuales también sería útil e interesante incluir al subgrupo de pacientes con criterios de exclusión para el presente estudio (pacientes con enfermedad neurológica previa y/o enfermedad de vasos de cuello).

\section{Conclusión}

En la población estudiada, no se ha detectado ningún ECDs. Teniendo en cuenta este punto, los hallazgos en las publicaciones previas y considerando que ningún evento neurológico catastrófico fue descrito en este tipo de procedimientos bajo anestesia regional y sedación, incluso en pacientes con factores de riesgo cardiovascular, consideramos que dicha modalidad debería ser utilizada siempre que sea posible. Respecto a la monitorización de la perfusión cerebral con tecnología NIRS, creemos resulta útil aunque no imprescindible bajo este protocolo anestésico, dada la incidencia casi nula de eventos. En consecuencia, cada centro debe tener en cuenta la relación costobeneficio de este tipo de monitorización. Dicha conclusión no abarca a las subpoblaciones excluidas en la presente investigación: pacientes con enfermedad neurológica previa y/o enfermedad de vasos de cueIlo (oclusión carotidea significativa y/o enfermedad de territorio vertebral). Tanto la utilidad de la monitorización con tecnología NIRS como el impacto de la estrategia anestésica empleada podrían ser objeto de análisis en futuros estudios en dicho subgrupo.

\section{Referencias}

1. Papadonikolakis $A$, Wiesler $E R$, Olympio MA, Poehling GG. Avoiding catastrophic complications of stroke and death related to shoulder surgery in the sitting position. Arthroscopy. 2008 Apr;24(4):481-2.

2. Pohl A, Cullen DJ. Cerebral ischemia during shoulder surgery in the upright position: a case series. J Clin Anesth. 2005 Sep;17(6):463-9.

3. Moerman AT, De Hert SG, Jacobs TF, De Wilde LF, Wouters PF. Cerebral oxygen desaturation during beach chair position. Eur J Anaesthesiol. 2012 Feb;29(2):82 7.

4. Casati A, Spreafico E, Putzu M, Fanelli G. New technology for noninvasive brain monitoring: continuous cerebral oximetry. Minerva Anestesiol. 2006 Jul-
Aug;72(7-8):605-25.

5. Tange $\mathrm{K}$, Kinoshita $\mathrm{H}$, Minonishi T, Hatakeyama N, Matsuda $\mathrm{N}$, Yamazaki M, et al. Cerebral oxygenation in the beach chair position before and during general anesthesia. Minerva Anestesiol. 2010 Jul;76(7):48590.

6. Murphy GS, Szokol JW, Marymont JH, Greenberg SB, Avram MJ, Vender JS, et al. Cerebral oxygen desaturation events assessed by near-infrared spectroscopy during shoulder arthroscopy in the beach chair and lateral decubitus positions. Anesth Analg. 2010 Aug;111(2):496505.

7. McCulloch TJ, Liyanagama K, Petchell J. Relative hypotension in the beach-chair position: effects on middle cerebral artery blood velocity. Anaesth Intensive Care. 2010 May;38(3):486-91.
8. Soeding PF, Wang J, Hoy G, Jarman $P$, Phillips $H$, Marks $P$, et al. The effect of the sitting upright or 'beachchair' position on cerebral blood flow during anaesthesia for shoulder surgery. Anaesth Intensive Care. 2011 May;39(3):440-8.

9. Jeong $\mathrm{H}$, Jeong $\mathrm{S}$, Lim $\mathrm{HJ}$, Lee J, Yoo KY. Cerebral oxygen saturation measured by near-infrared spectroscopy and jugular venous bulb oxygen saturation during arthroscopic shoulder surgery in beach chair position under sevoflurane-nitrous oxide or propofol-remifentanil anesthesia. Anesthesiology. 2012 May;116(5):1047-56.

10. Koh JL, Levin SD, Chehab EL, Murphy GS. Neer Award 2012: cerebral oxygenation in the beach chair position: a prospective study on the effect of general anesthesia compared with re- 
gional anesthesia and sedation. J Shoulder Elbow Surg. 2013 Oct;22(10):1325-31.

11. Yadeau JT, Liu SS, Bang $H$, Shaw PM, Wilfred SE, Shetty T, et al. Cerebral oximetry desaturation during shoulder surgery performed in a sitting position under regional anesthesia. Can J Anaesth. 2011 Nov;58(11):98692.
12. Aguirre J, et al. Cerebral oxygenation in patients undergoing shoulder surgery in beach chair position: comparing general to regional anesthesia and the impact on neurobehavioral outcome. Rev Esp Anestesiol Reanim. 2013:r

13. Murphy GS, Szokol JW. Blood pressure management during beach chair position shoulder surgery: what do we know? Can J Anaesth. 2011 Nov;58(11):97782.

14. Salazar D, Sears BW, Aghdasi B, Only A, Francois A, Tonino $P$, et al. Cerebral desaturation events during shoulder arthroscopy in the beach chair position: patient risk factors and neurocognitive effects. J Shoulder Elbow Surg. 2013 Sep;22(9):1228-35. 